regarding syphilis. A convenience sample was recruited from digital platforms (e.g., Facebook ${ }^{\circledast)}$ and bars, clinics, and community-based organization events.

Results Of 119 survey respondents, 90 (76\%) lived in the Anchorage/Mat-Su region. Of these, $10.0 \%$ (95\% confidence interval [CI]: 5.3-17.9) reported a syphilis diagnosis during the previous 12 months and having a median of 3 (interquartile range: 1-6) sex partners during the previous 6 months. High-risk behaviors commonly associated with syphilis were reported, including condomless anal sex $(36.7 \%$; 95\% CI: 27.4-47.0) during the previous month, $\geq 1$ episode of group sex $(26.7 \%$; 95\% CI: $18.6-36.6)$ during the previous 6 months, and meeting sex partners online or on a geospatial mobile app (66.7\%; 95\% CI: 56.4-75.5) during the previous 6 months. Additionally, 44.4\% (95\% CI: 34.6-54.7) reported a sex partner living outside Anchorage and 31.1\% (95\% CI: 22.5-41.3) outside Alaska.

Conclusion Large numbers of sex partners outside Alaska presents barriers to partner services among Anchorage MSM with syphilis. Opportunities include using innovative strategies (e.g., technology-based partner services and out-of-jurisdiction partnerships) to reach populations at risk for syphilis.

Disclosure No significant relationships.

\section{P501 LOW PREVALENCE OF HIGH-RISK ANAL HPV IN YOUNG GAY AND BISEXUAL MALES AFTER THE UNIVERSAL HPV VACCINATION PROGRAM IN AUSTRALIA}

${ }^{1}$ Eric Chow*, ${ }^{2}$ Sepehr Tabrizi, ${ }^{1}$ Christopher Fairley, ${ }^{1}$ Rebecca Wigan, ${ }^{2}$ Alyssa Cornall, ${ }^{2}$ Steph Atchison, ${ }^{2}$ Dorothy Machalek, ${ }^{3}$ Jane Hocking, 'Catriona Bradshaw, ${ }^{2}$ Suzanne Garland, ${ }^{1}$ Marcus Chen. ${ }^{1}$ Alfred Health, Melbourne Sexual Health Centre, Carlton, Australia; ${ }^{2} T$ The Royal Women's Hospital, Centre for Women's Infectious Disease Research, Parkville, Australia; ${ }^{3}$ University of Melbourne, Melbourne School of Population and Global Health, Parkville, Australia

\subsection{6/sextrans-2019-sti.582}

Background Australia introduced a school-based quadrivalent human papillomavirus (HPV) vaccination program for females in 2007. This was extended to include boys aged 12-13 from 2013 , with a two-year catch-up for boys aged $\leq 15$. This study examined HPV prevalence among young gay and bisexual males (GBM) who were age-eligible for vaccination in the school-based program.

Methods Males aged 16-20 years were recruited from sexual health clinics and the community in Melbourne in 20172018 , if they reported any form of male sexual contact, and were residents of Australia from 2013. A clinician-collected anal swab, self-collected penile swab and oral rinse were collected and analysed for detection and 37 HPV genotypes (Roche Linear Array). Preliminary results from 114 GBM were analysed and full results will be available for presentation.

Results The mean age of GBM was 18.6 years (SD 1.0). The majority $(80 \%)$ were recruited from clinics and 20\% from the community. The median number of lifetime male partners was 10 [IQR 5-25] for receptive oral sex, four [IQR 1-11] for receptive anal sex and one for insertive anal sex [IQR 0-6]. Overall, 64\% received at least one dose of vaccine documented via the National HPV Vaccination Program Register. Prevalence of quadrivalent vaccine-preventable HPV genotypes was 4.9\% (95\% CI: $1.6-11 \%$ ) for anal, 3.4\% (95\% CI: $0.7-$ $9.5 \%)$ for penile and $0 \%$ (95\% CI: $0-3.2 \%$ ) for oral sites. Only two men, both unvaccinated, had high-risk vaccine- preventable HPV genotypes: one with anal HPV16 (1\%); the other penile HPV16 (1\%).

Conclusion Statistical analysis comparing before and after the male vaccination program will be performed until recruitment is completed. The preliminary analysis shows the prevalence of anal HPV 16/18 among young GBM following the schoolbased male HPV vaccination was low. The addition of male HPV vaccination to female programs may reduce the incidence of anal cancer among GBM.

Disclosure No significant relationships.

\section{P503 TRENDS IN AWARENESS AND USE OF PREP AMONG HIV-NEGATIVE MEN WHO HAVE SEX WITH MEN IN VANCOUVER, TORONTO, AND MONTREAL}

${ }^{1}$ Heather Armstrong*, ${ }^{2}$ Shenyi Pan, ${ }^{1}$ Justin Barath, ${ }^{3}$ Gbolahan Olarewaju, ${ }^{4}$ Ricky Rodrigues, ${ }^{5}$ Marc Messier-Peet, ${ }^{5}$ Herak Apelian, ${ }^{2}$ Mark Hull, ${ }^{6}$ Darrell Tan, ${ }^{7}$ Nathan Lachowsky, ${ }^{8}$ Jody Jollimore, ${ }^{4}$ Trevor Hart, ${ }^{9}$ Daniel Grace, ${ }^{5}$ Gilles Lambert, ${ }^{10}$ Joseph Cox, ${ }^{3}$ David Moore. ${ }^{1} B C$ Centre for Excellence in HIVIAIDS, Epidemiology and Population Health, Vancouver, Canada; ${ }^{2}$ British Columbia Centre for Excellence in HIVIAIDS, Vancouver, Canada; ${ }^{3} B C$ Centre for Excellence in HIVIAIDS, Vancouver, Canada; ${ }^{4}$ Ryerson University, Toronto, Canada; ${ }^{5}$ Direction Régionale de Santé Publique Montréal, Montreal, Canada; ${ }^{6}$ St. Michael's Hospital, Centre for Urban Health Solutions, Li Ka Shing Knowledge Institute, Toronto, Canada; ' University of Victoria, School of Public Health and Social Policy, Victoria, Canada; ${ }^{8}$ Community Based Research Centre, Vancouver, Canada; ${ }^{9}$ University of Toronto, Toronto, Canada; ${ }^{10}$ McGill University, Montreal, Canada

\subsection{6/sextrans-2019-sti.583}

Background Awareness, availability, and public funding of preexposure prophylaxis (PrEP) has increased substantially since it was approved by Health Canada in 2016 but policies and guidelines vary by province. Given the dynamic nature of PrEP policy and promotion, we sought to determine awareness and use of PrEP among gay, bisexual, and other men who have sex with men (gbMSM) in Vancouver, Toronto, and Montreal.

Methods Beginning in $02 / 2017$, sexually-active gbMSM $\geq 16$ years and living in Vancouver, Toronto, or Montreal were recruited into a cohort study using respondent-driven sampling (RDS). Data are included up to $08 / 2018$. At study visits every 6 months (Vancouver) or 12 months (Toronto, Montreal), participants completed a computer-assisted self-interview which included questions on PrEP awareness and use in the past 6 months (P6M). We used RDS-adjusted, general estimating equations accounting for two levels of clustering (RDS recruitment chain; participant) to evaluate temporal trends (monthly prevalence) of awareness and P6M usage of PrEP among HIVnegative participants.

Results 1619 HIV-negative gbMSM completed 2074 study visits (1205 Montreal, 285 Toronto, 584 Vancouver). Over the course of the study, PrEP awareness significantly increased among gbMSM in Montreal from 62.6\% during the first 6month period to $84.8 \%$ during the last 6-month period (OR:1.07, 95\%CI:1.02-1.12, per month); awareness remained consistent in Toronto (89.8\%-96.0\%, ns) and Vancouver (84.5\%-95.0\%, ns). Use of PrEP increased significantly in all three sites: Montreal, $10.5 \%$ during the first 6-month period to $30.9 \%$ during the last 6-month period (OR:1.06, 95\% CI:1.02-1.11); Toronto, $12.5 \%$ to $23.3 \%$ (OR:1.15, 95\% CI:1.02-1.30); Vancouver, $11.4 \%$ to $35.2 \%$ (OR:1.16, $95 \%$ CI:1.06-1.27).

Conclusion Awareness of PrEP appears to be $\geq 85 \%$ among HIV-negative gbMSM across all three cities. Even though 
provincial policy and programs differ greatly in availability and cost of PrEP, use of PrEP increased in all sites during the course of the study.

Disclosure No significant relationships.

\section{P504 REDUCTION OF THE SPREAD OF HIV AMONG MEN WHO HAVE SEX WITH MEN IN BAMENDA-CAMEROON}

Assi Flaviurs. Vicony's Global Success World, Public Health, Bamenda, Cameroon

10.1136/sextrans-2019-sti.584

Background In 2010, i conducted a study that aimed to produce information on the magnitude and characteristics of the HIV epidemic in the MSM population of 18 years or more, and sexual behavior in two prison and region of the country between these region is Bamenda, located in the north west of the country with an estimated population of 2.5 million. Alternative Cameroon and Vicony's Global Success World (VGSW) in Bamenda was responsible for the execution of the study and was responsible for the protocol provided.

Methods

Data collection was carried out by the method of sampling conducted by respondents (Respondent Driven Sampling RDS) and the application of a serological survey anonymous linked. The national sample consisted of 200 MSM in the region of Bamenda by 333 .

Results HIV prevalence $8.1 \%$ in North West region (Ministry of public health 2010).

The $0 \%$ are considered bisexual, homosexual $75.2 \%, 1.8 \%$ heterosexual.

$10.8 \%$ of MSM in the region do not know where to go if they want to get tested for HIV.

21.9\% underwent a test for HIV in the last 12 months, and $50.3 \%$ received advice.

Only $10.6 \%$ knew that condoms can be achieved in health institutions.

28.1\% had to fake his sexual orientation.

Conclusion The results show a flaw in the processes of prevention and education for HIV and STIs among MSM and experience has served the Civil Society Organizations and the Government of the region to find a way to solve the problems that are evident in the response.

Clearly the stigma and discrimination in the area of HIV associated with MSM.

Bamenda could not be completed sample was estimated that 350 people (only reached 333) due to homophobia's attack project site and where material were destroyed, and tracking documents.

This study encourages us to move forward despite the adversities and difficulties.

Disclosure No significant relationships.

\section{P505 THE IMPACT OF GONORRHOEA VACCINATION IN MEN WHO HAVE SEX WITH MEN ON PREVALENCE AND RESISTANCE: MATHEMATICAL MODELLING STUDY}

\footnotetext{
${ }^{1}$ Janneke Heijne*, ${ }^{1}$ Maria Xiridou, ${ }^{2}$ Katy Turner, ${ }^{1}$ Birgit Van Benthem, ${ }^{3}$ Nicola Low. ${ }^{1}$ National Institute for Public Health and the Environment (RIVM), Epidemiology and Surveillance, Centre for Infectious Diseases Control, Bilthoven, Netherlands; ${ }^{2}$ University of Bristol, Bristol, UK; ${ }^{3}$ Institute of Social and Preventive Medicine (ISPM), Bern, Switzerland
}

10.1136/sextrans-2019-sti.585
Background Men who have sex with men (MSM) are disproportionately affected by Neisseria gonorrhoeae (gonorrhoea) and antimicrobial resistance (AMR) is common. Gonorrhoea vaccine development is challenging, but a N. meningitidis (group B) vaccine might have reduced gonorrhoea incidence by $\approx 30 \%$. This study aims to investigate the impact of vaccination on gonorrhoea transmission and on AMR in MSM.

Methods We developed a deterministic compartmental model of gonorrhoea transmission among Dutch MSM. We included three sexual activity classes that differed in numbers of partners/year and unprotected sex acts/partnership. AMR to firstline treatment was assumed to be complete, occurring at a low probability after treatment. We modelled a partially protective prophylactic vaccine (reducing susceptibility) providing 2 years' protection. We estimated the prevalence of sensitive and resistant gonorrhoea strains after 10 and 50 years for vaccine efficacies (VE) of 30\% (lower) and 60\% (higher) and different uptake levels in high sexual activity MSM.

Results Gonorrhoea prevalence in the model was $0.45 \%$ overall, $8.3 \%$ in high sexual activity MSM, and $0.002 \%$ resistant strains (baseline). With lower VE, after 10 years, the prevalence of the sensitive strain was reduced by $75 \%$ (for $50 \%$ uptake). AMR prevalence increased (irrespective of uptake) although less than without vaccination. With lower VE, after 50 years, AMR prevalence decreased only with uptake $\geq 60 \%$, levels at which the sensitive strain was already eliminated. With higher VE, AMR prevalence decreased after 10 years for uptake $\geq 30 \%$ and after 50 years for uptake of $\geq 20 \%$.

Conclusion In this modelling study, a partially protective prophylactic vaccine for gonorrhoea decreased overall prevalence, even with lower VE. This study also shows how vaccination can reduce the spread of AMR. For gonorrhoea, vaccination can decrease the speed at which AMR is transmitted, and even reduce AMR prevalence, but only with higher levels of vaccine efficacy or uptake in high sexual activity MSM.

Disclosure No significant relationships.

\section{P506 OPTIMIZING EPT FOR MSM AND TRANSGENDER WOMEN}

${ }^{1}$ Kristi Gamarel, ${ }^{2}$ Raha Richardson*, ${ }^{2}$ Rob Stephenson, ${ }^{2}$ Okeoma Mmeje. ${ }^{1}$ University of Michigan, Health Behavior and Health Education, Ann Arbor, USA; ${ }^{2}$ University of Michigan, Ann Arbor, USA

\section{$10.1136 /$ sextrans-2019-sti.586}

Background Despite increasing rates of HIV and other STIs among men who have sex with men (MSM) and transgender women (TW), expedited partner therapy (EPT) is not routinely offered to these priority populations. The goal of this study was to understand healthcare providers' (HCP) views and opinions regarding the use of EPT with MSM and TW. Methods Between May and October 2018, 18 HCP in Michigan completed semi-structured interviews. The interviews covered questions about knowledge, attitudes, experiences with, and barriers and strategies for delivering EPT to MSM and TW.

Results HCP $\left(\right.$ Mean $\left._{\mathrm{age}}=47.4\right)$ included MDs (33\%), NPs (61\%), RNs (5\%), and a Pharmacist (1\%). The majority of HCP (94.5\%) were willing to prescribe EPT for chlamydia and gonorrhea to MSM and TW. Several HCP in community health clinics were currently prescribing EPT to MSM and TW, whereas others followed CDC guidelines. Some HCP expressed concerns about the provision of EPT for individuals 